\title{
How guest molecules effect on the self-assembly of carboxylated pillar[5]arene in its complexes
}

\author{
H. Butkiewicz, S. Kosiorek, V. Sashuk, O. Danylyuk \\ Institute of Physical Chemistry Polish Academy of Sciences, Warsaw, Poland \\ hbutkiewicz@ichf.edu.pl
}

Carboxylated Pillar [5]arene (CPA5), first reported by Ogoshi in 2010 [1], is highly symmetrical pillar-shaped compound, composed of hydroquinone units linked by methylene bridges at the para-positions, modified by ten carboxylic acid groups. Its rigid hydrophobic, electron-rich cavity combined with its water solubility make it great candidate as host molecule for various electrondeficient guest or other neutral molecules. Moreover, carboxyl groups, that can take part in proton transfer, are located at the terminal positions of flexible aliphatic chains, so they can adjust to the size and shape of guests.

In 2015 Danylyuk described crystal self-assemby of CPA5 in its complex with ethanol molecules [2]. Authors described the chains of CPA5 molecules connected via cyclic carboxylic-carboxylic hydrogen bonds (HBs) as main supramolecular motif. Introduction of tertacaine guest reorganized the formation of HBs. Inspired by this result, we decided to investigate how guest molecules, decorated with different functional groups, effect on the self-assembly of the host.

Here we want to present our results on the X-ray structures of CPA5 in the form of its host-guest complexes with viologens, guanidine and amidine compounds. Our study on the CPA5-viologens complexes shows that the main chain motif is dictated by very strong carboxylic-carboxylate HBs [3]. Altering the guest into an amidine or guanidine molecules changes main synthon into amidinium-carboxyl/ate and guanidinium-carboxyl/ate HBs. In a broader perspective our results may have potential applications in drug delivery and molecular recognition systems.

[1] Ogoshi, T., Hashizume, M., Yamagishi, T., Nakamoto, Y. (2010). Chem Comm, 21, 3708.

[2] Danylyuk, O., Sashuk, V. (2015). CrystEngComm, 17, 719.

[3] Butkiewicz, H., Kosiorek, S., Sashuk, V., Danylyuk, O. (2021). CrystEngComm, 23, 1075.

Keywords: host-guest complexes; macrocycle; pillar[n]arene 